\title{
A corrigendum to "A note on compact-like semitopological groups" [Carpathian Math. Publ. 2019, 11 (2), 442-452]
}

\section{Peng Liang-Xue}

School of Mathematics, Faculty of Science, Beijing University of Technology, Beijing 100124, China E-mail: pengliangxue@bjut.edu.cn

Recall that a topological space $X$ is weakly semiregular, if $X$ has a base consisting of regular open sets, that is such sets $U$ that $U=\operatorname{int} \bar{U}$. In [1] is stated the following result.

Lemma 3 ([1]). Let $(X, \tau)$ be a weakly semiregular space, $(Y, \sigma)$ be a space and $\pi: X \rightarrow Y$ be a continuous clopen surjection. Then $Y$ is a weakly semiregular space.

Unfortunately, lemma's proof from [1] contains an error. Namely, the inclusion $\pi(\bar{U}) \subset$ $\pi \pi^{-1}(V)$ fails, for instance, when $\pi$ is the identity map and $U=V$ is any regular open set such that $U \neq \bar{U}$.

Fortunately, in the paper [1] Lemma 3 is applied only once, namely in conjunction with Lemma 1 to prove Proposition 2. This application can be fixed because the map $\pi$ considered in Lemma 1 satisfies a condition $\pi^{-1}(\pi(U))=U$ for every regular open subset of $X$. Adding this condition to Lemma 3, we can derive the required conclusion as follows.

Let $y \in Y$ be any point and $V \in \sigma$ be any open neighborhood of $y$. Pick a point $x \in \pi^{-1}(y)$. Since $\pi^{-1}(V)$ is an open neighborhood of $x$ and $X$ is weakly semiregular, there exists a regular open subset $U$ of $X$ such that $x \in U \subset \pi^{-1}(V)$. Since the mapping $\pi$ is continuous and clopen, we have $\pi(\bar{U})=\overline{\pi(U)}$ and $\pi(U)$ is open in $Y$. Since $U$ is open, the set $X \backslash \bar{U}$ is a regular open subset of $X$. Then $X \backslash \bar{U}=\pi^{-1}(\pi(X \backslash \bar{U}))$. Thus $\bar{U}=\pi^{-1}(\overline{\pi(U)})$.

Suppose that there exists a point $z \in \operatorname{int} \overline{\pi(U)} \backslash V$. Then $\pi^{-1}(z) \subset X \backslash \pi^{-1}(V)$ and $\pi^{-1}(z) \subset \pi^{-1}$ (int $\left.\overline{\pi(U)}\right) \subset \pi^{-1}(\pi(\bar{U}))=\bar{U}$. This contradicts with int $\bar{U} \subset \pi^{-1}(V)$. Thus $y \in \operatorname{int} \overline{\pi(U)} \subset V$, and hence $Y$ is a weakly semiregular space.

\section{References}

[1] Ravsky A. A note on compact-like semitopological groups. Carpathian Math. Publ. 2019, 11 (2), $442-452$. doi:10.15330/cmp.11.2.442-452

Received 25.10.2021

$\mathrm{y} \Delta \mathrm{K} 12.546 .82,512.546 .8,512.546$

2020 Mathematics Subject Classification: 22A15, 54H99, 54H11.

The author is supported by Beijing Natural Science Foundation (Grant No. 1202003) and the National Natural Science Foundation of China (Grant No. 12171015, 11771029) 